$$
\text { "murillo" — 2008/9/1 — 17:25 — page } 43 \text { — \#1 }
$$

\title{
Analyse d'obstacles lies a la notion de fonction reciproque
}

\author{
Susana Murillo-López et André Antibi
}

\begin{abstract}
L'article présente une réflexion sur les difficultés éventuelles des étudiants au cours de l'étude des fonctions réciproques. Nous nous intéressons à l'enseignement de cette notion dans le cadre de la transition entre le lycée et la première année d'université en France. L'article résume la façon de présenter de nos jours le concept de fonction réciproque dans le curriculum français. Nous présentons des propositions d'enseignement issues de quelques travaux de recherche anglophones. Cela nous permet de mettre en lumière l'existence d'obstacles dans l'apprentissage et de difficultés dans l'enseignement de cette notion.
\end{abstract}

Key words and phrases: fonction réciproque, transition lycée-université, obstacle didactique, dé-transposition.

ZDM Subject Classification: A05, I95.

\section{Introduction}

Lors de son enseignement d'analyse au niveau L1 français, André Antibi a repéré chez des étudiants des difficultés liées à la notion de fonction réciproque. Ce constat nous a conduit à nous intéresser à cette notion, dont le statut a beaucoup évolué dans l'enseignement des mathématiques en France. En effet, actuellement il n'est étudié qu'au niveau post-secondaire, car au lycée il est maintenant seulement implicite et au choix du professeur.

Nous avons choisi de placer nos travaux dans le cadre de la transition entre la Terminale (dernière année de lycée) et la première année universitaire.

Copyright (c) 2008 by University of Debrecen 


$$
\text { "murillo" — 2008/9/1 — 17:25 — page } 44 \text { — \#2 }
$$

Nous précisons d'abord certains aspects mathématiques de cette notion. Nous effectuons une analyse d'obstacles didactiques possibles entraînant des difficultés pour les étudiants, en tenant compte du curriculum français actuel et de certains travaux de recherche issus du milieu anglophone. Enfin, nous terminons par quelques suggestions.

\section{La notion de fonction réciproque}

Avant d'aborder la notion de fonction réciproque nous revenons sur la notion de fonction. Puis nous préciserons différentes propositions de définition de la notion de fonction réciproque de nos jours.

\subsection{Précisions sur la notion de fonction}

La rigueur de l'époque des Mathématiques Modernes a marqué une distinction pour beaucoup de mathématiciens entre la définition de fonction et d'application. En général la distinction est très particulière; parfois elle n'est qu'une commodité de langage qui permet d'éviter certaines confusions et qui selon M. Rogalski (2001) n'a rien d'obligatoire. De son côté, l'auteur emploie le mot fonction pour travailler sur des «fonctions d'une ou plusieurs variables réelles », et celui d'application «soit lorsque les ensembles qui interviennent sont a priori quelconques, soit lorsque l'un d'eux au moins est un espace de 'fonctions numériques' 》(Rogalski, 2001, p. 20). La définition donnée par L. Schwartz dans son cours d'analyse de 1981, qui constitue pour certains une référence indiscutable d'un point de vue théorique, souligne le fait qu'il n'y a pas de distinction entre les termes « fonction » et « application » :

«Soit $I$ et $J$ deux ensembles, on appelle application de $I$ dans $J$ ou fonction définie sur $I$ à valeurs dans $J$ toute correspondance $f$ qui, à chaque élément $x$ de $I$, fait correspondre un élément, noté $f(x)$, de $J$. La notation $I \stackrel{f}{\rightarrow} J$ signifie que $f$ est une application ou fonction de $I$ dans $J$. $I$ s'appelle ensemble initial, $J$ ensemble final de l'application » (Schwartz, 1981, p. 6). 


$$
\text { "murillo" — 2008/9/1 — 17:25 — page } 45 \text { — \#3 }
$$

\subsection{La notion de fonction réciproque}

Elle est présente dès le début du XVIII ${ }^{\mathrm{e}}$ siècle $^{1}$, bien que de manière intuitive. C'est le cas si l'on pense à l'idée d'inversion comme un processus : on peut faire appel aux premières préoccupations techniques des analystes autour de la différentiation et des quadratures (la dérivation et l'intégration).

Les mathématiciens de la fin du $\mathrm{XX}^{\mathrm{e}}$ siècle proposent des définitions de la notion de fonction réciproque liées à la notion de bijection de manière intrinsèque au secteur des fonctions ou en liant le point de vue des fonctions avec celui des équations.

\subsubsection{La proposition de L. Schwartz intrinsèque au secteur des fonctions}

Quand on a une fonction $f: I \rightarrow J$, qui fait correspondre à tout élément $x$ de $I$ un élément $y$ de $J$, assez vite se pose la question des conditions qui nous permettent d'affirmer qu'il existe une autre fonction $g$ qui à $y$ fasse correspondre $x$; c'est-à-dire, la fonction qui fasse «le chemin de retour » qui est nommé fonction réciproque.

A ce sujet, L. Schwartz (1981) propose la définition suivante :

« Soit $f$ une bijection, et soit $y \in J$. Appelons $f^{-1}(y)$ l'unique élément $x$ de $I$ tel que $f(x)=y$. Nous venons de définir une application $f^{-1}$ de $J$ dans $I$. C'est encore une bijection, on dit que c'est l'application

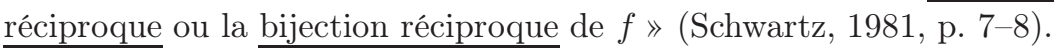

\subsubsection{La proposition de M. Rogalski liant deux points de vue}

M. Rogalski (2001) propose la définition suivante à partir de l'équation qui est attachée à l'application :

«Soit $f: I \rightarrow J$ une application, et $y$ un élément de $J$. On dit qu'on veut résoudre l'équation $\left(e_{f, y}\right)$, et on note $\left(e_{f, y}\right): f(x)=y$, lorsqu'on recherche un élément $x$ de $I$ dont l'image par $f$ est $y$ (on peut aussi dire qu'on recherche un antécédent $x$ de $y$ ). On dit que $x$ est l'inconnue, et que $y$ est donné. Un élément $x$ de $I$ qui répond à la question est dit une solution de l'équation » (Rogalski, 2001, p. 18).

Le fait de disposer de points de vue différents peut nous être utile en mathématiques. Ainsi, pour résumer les notions d'injection, surjection et bijection, M. (1984).

1. Pour plus de détails consulter par exemple : A-P. Youschkevitch (1981) ou bien J. Itard 


$$
\text { "murillo" — 2008/9/1 — 17:25 — page } 46 \text { — \#4 }
$$

Rogalski (2001) comme d'autres auteurs, ajoute au langage usuel et au point de vue formel, celui des équations et le cas du graphe des fonctions numériques. Il définit ainsi la fonction réciproque :

«... si l'application $f: I \rightarrow J$ est bijective, alors tout élément $x$ de $J$ est l'image par $f$ de son unique antécédent $y$ dans $I$, donc bien déterminé par $x$; la correspondance $x \rightarrow y$ ainsi définie est une application de $J$ en $I$, appelée application réciproque de $f$ et notée $f^{-1}$. Par définition, on a l'équivalence :

$$
\left.\begin{array}{l}
y=f^{-1}(x) \\
x \in J
\end{array}\right\} \Leftrightarrow\left\{\begin{array}{l}
x=f(y) \\
y \in I
\end{array} 》\right.
$$

(Rogalski, 2001, p. 22).

La proposition intrinsèque au secteur fonctionnel et la proposition liant le point de vue fonctionnel à celui des équations ne sont pas enseignées intégralement dans le secondaire comme nous allons le rappeler dans le paragraphe 2.1.

\section{L'enseignement de la fonction réciproque}

Ce paragraphe rappelle ce qui est proposé dans les programmes de 2002 pour l'enseignement de l'analyse en Terminale $S$, avec une attention particulière sur ce qui est retenu par les Instructions Officielles à propos de la notion de fonction réciproque. Puis, il fait état de quelques résultats de travaux de recherche et de quelques réflexions générales à propos de cette notion et de son enseignement.

\subsection{Quelques précisions sur les programmes français}

Le statut du concept de fonction réciproque dans l'enseignement français a beaucoup évolué depuis le début du XXe siècle. M. Artigue (1996 et 2003) et P. Trabal (1997) exposent l'évolution de l'enseignement de l'analyse au lycée ${ }^{2}$. Après un grand formalisme à l'époque des mathématiques modernes, la fin du XXe siècle voit apparaître un constructivisme mal compris, marqué par une opposition entre l'apprentissage de concepts et l'apprentissage de techniques (Artigue, 2003). Cela a entrainé une situation de crise qui n'est pas un phénomène isolé et qui a conduit à réserver les ambitions théoriques aux enseignements universitaires.

2. Pour plus de détails le lecteur pourra se référer à ces textes. 


$$
\text { "murillo" — 2008/9/1 — 17:25 — page } 47 \text { — \#5 }
$$

Dans le cas du chapitre d' « Analyse » du programme de Terminale S de l'année 2002 (enseignement obligatoire), les prescriptions institutionnelles n'incluent pas l'étude des fonctions réciproques. Elles proposent l'étude des fonctions logarithme et exponentielle, mais n'imposent pas les modalités d'introduction de ces fonctions. Le caractère bijectif et réciproque ne figure pas dans le programme.

La seule précision donnée dans les premiers commentaires généraux du programme de Terminale $\mathrm{S}$ est la nécessité d'introduire la fonction exponentielle très tôt dans l'année. Ceci suppose d'aborder la fonction exponentielle avant la fonction logarithme.

Plus particulièrement, dans le chapitre consacré à l'Analyse deux titres nous intéressent : «Introduction de la fonction exponentielle » et «Etude des fonctions logarithmes et exponentielles », car c'est un des cas les plus classiques de fonctions étudié au lycée où on pourrait traiter la notion de fonction réciproque.

Dans le premier, on s'aperçoit que l'introduction de la fonction exponentielle part d'un problème concret pour l'élève, celui de la radioactivité traité en physique, ou bien de la recherche des fonctions $f$ dérivables sur $\mathbb{R}$ telles que $f(x+y)=f(x) f(y)$.

Dans le second, on repère trois possibilités pour l'introduction de la fonction logarithme : partir des propriétés des fonctions exponentielles; poser le problème des fonctions $f$ dérivables sur $\mathbb{R}^{+*}$ telles que $f(x y)=f(x)+f(y)$ et admettre l'existence de primitives pour la fonction $x \mapsto 1 / x$ lorsque $x>0$; ou traiter le logarithme après l'intégration. Il y a une différence entre la première proposition et les deux autres : dans celle là il est nécessaire d'étudier auparavant la fonction exponentielle, ce qui entrainerait le fait d'aborder au moins de manière implicite la notion de fonction réciproque. Pour les deux autres, il faut avoir admis au moins que les primitives pour la fonction $x \mapsto 1 / x$ pour $x>0$ existent; les fonctions exponentielle et logarithme seraient donc introduites indépendamment l'une de l'autre.

Ainsi, le choix de la manière d'introduire la fonction logarithme est lié à l'ordre des contenus et peut nécessiter de disposer de la notion de fonction réciproque. Les courbes des fonctions $x \mapsto e^{x}$ et $x \mapsto \ln x$ seront représentées graphiquement, mais il n'est dit nulle part qu'elles seront comparées à la première bissectrice dans un repère orthonormé pour préciser la symétrie de l'une par rapport à l'autre.

Les professeurs du niveau universitaire ont eux, une liberté assez grande dans le cadre de leur cours d'analyse. Ils peuvent décider d'inclure un paragraphe spécifique sur la notion de fonction réciproque. 


$$
\text { "murillo" — 2008/9/1 — 17:25 — page } 48 \text { — \#6 }
$$

Ainsi, lorsqu'un étudiant arrive en première année d'université, il peut avoir des difficultés à propos de la fonction réciproque ou du caractère bijectif d'une fonction : il se peut que pour lui les concepts de fonction réciproque et de bijection ne soient ni disponibles ni mobilisables (Robert, 1998). En effet, au lycée, ces notions ont parfois été traitées d'une manière implicite, voire pas du tout.

\subsection{Quelques travaux de recherche sur la fonction réciproque}

Les résultats des travaux que nous prenons en compte ici sont issus du milieu anglophone, en l'absence de travaux récents à propos de l'enseignement et/ou l'apprentissage de la notion de fonction réciproque dans le milieu francophone. Nous avons porté notre attention sur les références suivantes, non exhaustives : R. Even (1992), D. Vidakovic (1996), C. Lucus (2006) et I. Bayazit et E. Gray (2004).

\subsubsection{La notion de « défaire » (undoing) de R. Even}

Nous avons décidé de traduire « undoing » par « défaisant».

Dans son travail, R. Even émet l'hypothèse que considérer la fonction réciproque comme « défaisant »ce que la fonction « fait», aide à la compréhension du concept de fonction réciproque. Pour l'auteur, la notion de « défaire » est une idée informelle de la fonction réciproque, mais qui capture l'essence de la définition. Un des résultats a été que presque la moitié des futurs enseignants semblent ignorer ou ne pas prendre en compte cette signification de la fonction réciproque; ce qui est illustré par la tendance à faire tous les calculs requis pour trouver $\left(f^{-1} \circ f\right)(512,5)$ pour une fonction bijective donnée, au lieu d'utiliser la signification conceptuelle de la fonction réciproque selon laquelle on peut dire sans aucun calcul que le résultat est 512,5.

Cependant, dans ses résultats elle écrit qu'il y a un risque à limiter la conception de fonction réciproque à cette conception 《naïve » de 《 défaire », car il pourrait en résulter quelques difficultés : il est possible d'arriver à penser à tort, que toutes les fonctions ont une fonction réciproque. En outre, l'imprécision de l'idée de « défaire » lorsque les fonctions sont plus complexes peut engendrer des confusions entre des fonctions, par exemple entre $f(x)=x^{3}$ et $g(x)=3^{x}$, et $/$ ou entre $f^{-1}(x)=(x)^{1 / 3}$ et $g^{-1}(x)=\log _{3} x$. Toujours d'après R. Even il semblerait que les difficultés de compréhension de la nature de ces quatre fonctions particulières soient liées à l'absence d'une bonne compréhension de la fonction réciproque. 


$$
\text { "murillo" — 2008/9/1 — 17:25 — page } 49-\# 7
$$

R. Even conclut que le fait de percevoir la fonction réciproque comme « défaisant » est puissant, mais pas assez pour traiter tous les aspects du concept de fonction réciproque. Pour cela, il faudrait avoir deux meilleures connections entre connaissances procédurales et conceptuelles. En effet, elle affirme qu'en général, la compréhension d'une idée inclut les deux types de connaissances : les procédurales et les conceptuelles, ainsi que les relations entre elles, car lorsque concepts et procédures ne sont pas connectés, on peut avoir une bonne intuition mais ne pas être capable de résoudre des problèmes, ou bien, générer des réponses mais ne pas comprendre ce qu'on a fait. Sur ce point R. Even semble rejoindre les développements de R. Douady, auteur des aspects outil ou objet d'un même concept mathématique (Douady, 1986).

$\underline{\text { Remarque }}$ : L'idée intuitive de 《défaire » est en cohérence avec la notion de fonction multiforme (voir paragraphe 3.2). Cette idée est efficace si $f$ est une bijection, mais dangereuse si $f$ n'est pas une bijection.

\subsubsection{Au niveau universitaire (D. Vidakovic; C. Lucus)}

L'article de D. Vidakovic se focalise sur un objectif : « découvrir » la manière dont le concept de fonction réciproque peut être appris. Pour cela l'auteur cherche une décomposition génétique du concept de fonction réciproque. Une décomposition génétique d'un concept mathématique consiste en une description des méthodes de constructions mentales pour développer un schéma de ce concept.

D. Vidakovic construit une version «préliminaire»d'une décomposition génétique du concept de fonction réciproque pour la proposer à l'enseignement au niveau universitaire. Elle décrit la succession d'étapes que les étudiants doivent franchir pour apprendre le concept mathématique de fonction réciproque (Vidakovic, 1996, p. 305) :

(1) Avoir déjà développé un processus ou un objet pour le concept de fonction.

(2) Coordonner deux fonctions ou plus en tant que processus, pour définir la composition de deux fonctions, notée $h=f \circ g$.

(3) Utiliser le schéma d'une fonction construit précédemment et la composition de fonctions pour définir la fonction réciproque.

(4) Comprendre et appliquer le processus inverse pour des situations particulières. 


$$
\text { "murillo" — 2008/9/1 — 17:25 — page 50 — \#8 }
$$

C. Lucus s'intéresse, lui, aux connaissances d'enseignants ordinaires ${ }^{3}$ sur les acquis dont leurs étudiants ont besoin pour travailler sur la composition de fonctions et sur l'opération elle-même. Ceux qui ont participé à la recherche de C. Lucus donnent leurs opinions sur ce qu'ils pensent nécessaire aux étudiants pour commencer l'étude de la composition des fonctions. De leurs points de vue, ils ont besoin d'être à l'aise avec la manipulation d'expressions algébriques, de savoir la définition de fonction ainsi que différentes représentations de ce concept et de pouvoir se servir de graphes dans le plan cartésien. En ce qui concerne l'enseignement de l'opération « composition de fonctions », les professeurs disent qu'il est nécessaire d'insister sur le concept de fonction avec ses ensembles de définition et de faciliter les calculs algébriques.

La décomposition génétique proposée par D. Vidakovic ainsi que les résultats des déclarations des enseignants participant à l'étude de C. Lucus sont relativement standards en France. Les travaux de R. Even, D. Vidakovic et C. Lucus sont issus du champ de la psychologie. Les constructions théoriques qu'ils proposent pour analyser des phénomènes d'enseignement et d'apprentissage semblent éloignées des théories de didactique des mathématiques françaises.

Dans les articles considérés, les auteurs n'indiquent pas les conséquences éventuelles de cet enseignement sur l'apprentissage des étudiants. En revanche dans les travaux de I. Bayazit et E. Gray, relatés ci-dessous, nous trouvons des conclusions par rapport à ces conséquences après deux enseignements différents.

2.2.3. L'influence de l'enseignement sur l'apprentissage (I. Bayazit et E. Gray)

I. Bayazit et E. Gray étudient deux enseignements ordinaires différents en Turquie (deux enseignants de lycée avec 24 et 25 ans d'ancienneté et deux groupes de Seconde $-9^{\text {th }}$ grade - ). Leur article essaie d'expliquer la relation entre les pratiques d'enseignants et l'apprentissage des élèves dans le contexte des fonctions et en particulier pour comprendre les fonctions réciproques.

- L'un a centré son enseignement sur la notion de « défaire » et son premier objectif a été de fortifier la compréhension des élèves sur la condition de bijection avant l'exposition formelle; il a créé des connections entre idées ainsi que entre représentations. Il s'est servi de diagrammes de Venn, d'ensembles de couples ordonnés, de graphes et d'expressions algébriques. Pour

3. Enseignant ordinaire : celui qui n'a pas de lien avec la recherche en didactique des mathématiques. 


$$
\text { "murillo" — 2008/9/1 — 17:25 — page } 51 \text { — \#9 }
$$

lui, les expressions algébriques, en particulier linéaires, ne sont pas productives pour expliquer l'essentiel d'une fonction réciproque, mais il a utilisé les formes algébriques de fonctions linéaires pour expliquer l'inversion d'opérations.

- Pour le deuxième, l'enseignement a été focalisé sur des habiletés algorithmiques et des acquisitions de règles de procédure. Il a utilisé les connaissances précédentes des élèves et a montré des analogies avec des situations de la vie quotidienne pour encourager l'acquisition de ces habiletés de procédure chez les élèves. Il n'a intégré dans son enseignement ni les représentations graphiques cartésiennes ni les ensembles de couples, mais un exemple avec les diagrammes de Venn pour expliquer la nécessité de la condition de bijection.

A l'issue de ces deux enseignements, les élèves sont testés. Les auteurs décrivent les erreurs des élèves à deux questions posées : l'une pour tester la compréhension de la notion de «défaire » et la propriété de bijection, l'autre pour observer l'utilisation du concept de fonction réciproque dans une situation graphique.

Les résultats de leur enquète les amène à présenter dans leur conclusion les points suivants :

- La manière de présenter une notion par l'enseignant joue un rôle important dans l'apprentissage des élèves.

- Concernant la notion de fonction réciproque, pour lui donner du sens, il ne suffit pas de s'appuyer sur les pré-requis des étudiants (les opérations algébriques par exemple) ou sur des analogies avec la vie de tous les jours. Il conviendrait de s'appuyer sur des représentations variées, en mettant en évidence des liens entre la notion de fonction réciproque et les notions d'injectivité et de surjectivité.

\subsection{Quelques exemples de fonctions et leurs réciproques dans la transition lycée/université}

Inspirés par les résultats de ces travaux, nous parlerons dans cette section des difficultés qui peuvent se présenter lors de l'apprentissage de la fonction réciproque. Nous reprenons la caractéristique de symétrie de deux fonctions réciproques par rapport à la droite d'équation $y=x$ dans un repère orthonormé, la définition plus générale de fonction réciproque et les choix par rapport à la définition de certaines fonctions : exponentielle et logarithme, carrée et racine carrée et quelques fonctions trigonométriques. 


\subsubsection{Cas de fonctions de $\mathbb{R}$ dans $\mathbb{R}$}

Par rapport aux applications composées, si on a une fonction $f: I \rightarrow J$ bijective, on sait que le résultat de la composition de la fonction avec sa bijection réciproque sera l'identité; c'est-à-dire, on peut affirmer que $\forall y \in J$ on a $\left(f \circ f^{-1}\right)(y)=y$ et que $\forall x \in I$ on a $\left(f^{-1} \circ f\right)(x)=x$. Ainsi, pour une fonction $f$ bijective définie de $\mathbb{R}$ dans $\mathbb{R}$, les courbes représentatives des fonctions $f$ et $f^{-1}$ dans un même repère orthonormé sont symétriques par rapport à la droite d'équation $y=x$. Cette caractéristique pourrait être mise en relation avec l'idée de « défaire » mentionnée plus haut.

Cependant, il peut y avoir plusieurs sources d'obstacles pour les étudiants à partir de cette caractéristique de symétrie des deux courbes représentatives des fonctions $f$ et $f^{-1}$ par rapport à la première bissectrice dans un repère orthonormé :

- Il faut préciser que c'est une particularité des fonctions bijectives définies de $\mathbb{R}$ dans $\mathbb{R}$. Pour des fonctions définies dans des ensembles plus généraux, $\mathbb{R}^{2}$ par exemple, on ne pourrait plus représenter un « échange » d'axes entre l'ensemble de définition de $f$ et l'ensemble de définition de $f^{-1}$ pour que cette symétrie soit visible.

- Un autre aspect réside dans la particularité que ce soit dans un repère orthonormé, car dans un autre repère cette symétrie ne se réalise pas.

\subsubsection{De manière plus générale}

Lorsqu'on travaille avec une partie des ensembles $I$ et $J$, on a des situations plus générales par rapport aux définitions données auparavant; par exemple, la définition d'une image réciproque par une application existe même si la fonction ou l'application donnée n'est pas bijective. Cette situation est montrée par L. Schwartz :

« Soit $A$ une partie de $I$ et $B$ une partie de $J$. On appelle $f^{-1}(B)$ la partie de $I$ formée de tous les $x$ tels que $f(x) \in B$. Evidemment $f^{-1}(\emptyset)=\emptyset$. Avec une notation abrégée, on a $f^{-1}(B)=\{x \mid f(x) \in B\}$ et $f(A)=$ $\{f(x) \mid x \in A\}$. On peut aussi avoir $f^{-1}(B)=\emptyset$ pour $B \neq \emptyset$. Par exemple, si $f$ est l'application $x \mapsto x^{2}$ de $\mathbb{R}$ dans $\mathbb{R}, f^{-1}(\{-1\})=\emptyset$.

On remarque donc que l'application $\left[^{-1}\right.$ ainsi définie est plus simple ${ }^{4}$ que l'application $f^{-1}$ définie plus haut. La partie $f^{-1}(B)$ s'appelle image réciproque de $B$ par l'application $f$. Il y a lieu de remarquer que cette

4. «Plus simple» car elle existe dans tous les cas, que $f$ soit bijective ou non. 


$$
\text { "murillo" — 2008/9/1 — 17:25 — page 53 — \#11 }
$$

définition ne suppose nullement que $f$ soit bijective. De toute façon, si $y \in J$, on a le droit de parler de $f^{-1}(\{y\})$, mais c'est une partie de $I$ et non un élément de $I$. Elle peut comprendre plus d'un élément, si $f$ n'est pas injective, et elle peut être la partie vide, si $f$ n'est pas surjective. Si $f$ est bijective, on a exactement $f^{-1}(\{y\})=\left\{f^{-1}(y)\right\} »$ (L. Schwartz, 1981, p. 8-9).

Cette généralisation pourrait faciliter la compréhension de la notion de fonction réciproque, la fonction donnée ne nécessitant aucune restriction des domaines de définition pour garantir l'existence de sa fonction réciproque. Cependant, elle peut être également une source d'obstacles à cause de sa notation. On peut remarquer qu'une écriture différente convient mieux pour se référer à la partie $f^{-1}(B)$, telle que ${ }^{-1}(B)$, car il peut y avoir confusion avec la fonction qui requiert le caractère bijectif pour admettre la fonction réciproque.

En effet, toutes les restrictions ou conventions effectuées sur les ensembles de définition de certaines fonctions pour garantir leur bijection, peuvent constituer des difficultés d'apprentissage de la fonction réciproque pour les étudiants. Nous développons ci-dessous quelques exemples de fonctions présentes dans la transition du lycée à l'université.

\subsubsection{Les fonctions bijectives par définition}

Lorsqu'au lycée on mentionne la fonction exponentielle et logarithme, on pourrait aborder le concept de fonction réciproque; si on ne le fait pas c'est peut-être parce qu'on n'a pas dans le programme toutes les notions d'injection, de surjection et de bijection.

L'un des choix donnés pour introduire la fonction logarithme est de partir des propriétés de la fonction exponentielle : la notion de fonction réciproque serait donc abordée au moins implicitement.

Un autre choix serait de partir des propriétés de la fonction logarithme pour introduire la fonction exponentielle. Cependant, cela impliquerait le retour aux anciennes instructions officielles, car il serait nécessaire de commencer par la fonction logarithme et de la définir comme une primitive de la fonction inverse.

Lors de la résolution d'équations exponentielles ou logarithmiques, ce statut implicite de la notion de fonction réciproque est aussi présent. Bien que les instructions officielles n'imposent pas ce type de raisonnement, l'enseignement met souvent en évidence le caractère réciproque entre ces deux fonctions. Dans ce cas, il n'est pas nécessaire de faire des restrictions sur leurs ensembles de définition. 
Il en est ainsi, par exemple, des formules : $e^{\ln x}=x($ pour $x>0)$ et $\ln \left(e^{x}\right)=x$ (pour tout $x$ ).

Dans le cas général, comme on l'a vu ci-dessus, la notion de fonction réciproque peut être étroitement liée à la résolution d'une équation.

\subsubsection{La restriction des domaines de définition}

Le cas des fonctions carrée et racine carrée pourrait constituer un bon exemple où la restriction des domaines de définition est nécessaire. On pourrait alors reprendre cet exemple au niveau universitaire pour aborder cette notion, les fonctions carré et racine carrée étant déjà connues par les étudiants. Or, pratiquement ces deux fonctions, carré et racine carrée, sont souvent introduites de façon indépendante, à des moments distincts du cursus scolaire.

Cet exemple est à la fois simple et riche.

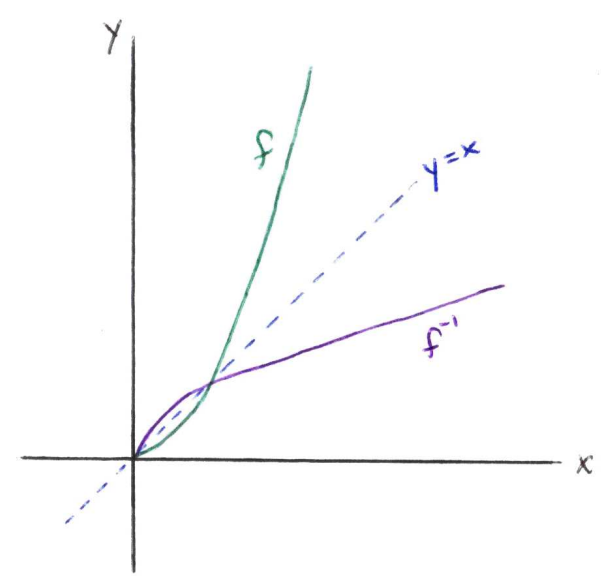

Figure 1

En effet, la définition de la fonction $f: x \mapsto x^{2}$ bijective de $\mathbb{R}^{+}$dans $\mathbb{R}^{+}$et respectivement, sa fonction réciproque $f^{-1}: x \mapsto \sqrt{x}$ de $\mathbb{R}^{+}$dans $\mathbb{R}^{+}$(Figure 1 ) sert à illustrer trois aspects importants : la symétrie entre deux fonctions réciproques par rapport à la première bissectrice dans un repère orthonormé; le besoin de restreindre le domaine de définition d'une fonction donnée pour vérifier la bijection et pour qu'elle admette sa réciproque; et enfin, la convention mathématique pour cette restriction de domaine, qui doit être acceptée comme telle. 
On rencontre les mêmes difficultés dans l'étude des fonctions trigonométriques réciproques. On trouve une situation analogue de convention sur les ensembles de définition de la fonction sinus, pour définir sa fonction bijective réciproque Arcsinus.

Ainsi, on établit la convention de restreindre le domaine de définition à l'intervalle $\left[-\frac{\pi}{2} ; \frac{\pi}{2}\right]$ pour que la fonction $f: x \mapsto \sin x$ de $\left[-\frac{\pi}{2} ; \frac{\pi}{2}\right]$ dans $[-1 ; 1]$ soit bijective, et pour définir en conséquence sa bijection réciproque $f^{-1}: x \mapsto \arcsin x$ de $[-1 ; 1]$ dans $\left[-\frac{\pi}{2} ; \frac{\pi}{2}\right]$.

\subsubsection{Les fonctions $f \circ f^{-1}$ et $f^{-1} \circ f$}

Dans le cas où $f$ est bijective, les relations $\left(f^{-1} \circ f\right)(x)=x$ et $\left(f \circ f^{-1}\right)(x)=x$ sont intuitives.

Dans le cas où $f$ n'est pas bijective, il n'en est pas de même.

Un exemple: cas de la fonction $f: x \mapsto \sin x$.

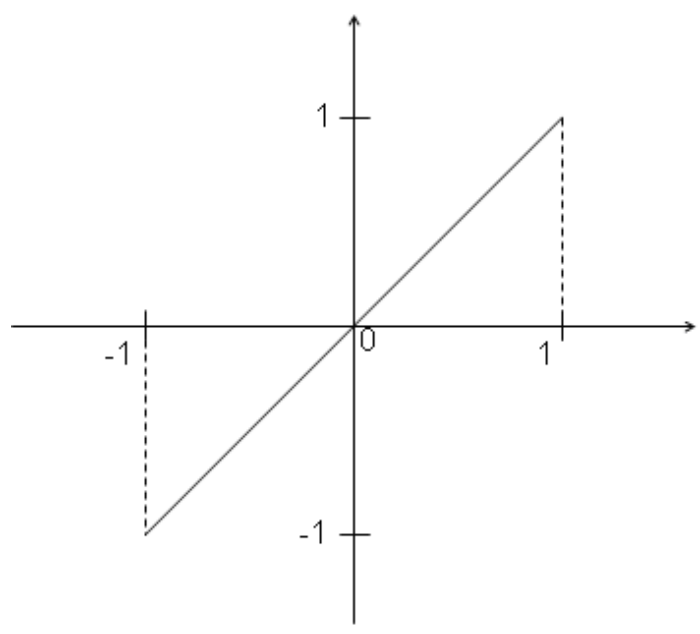

Figure 2. $\left(f \circ f^{-1}\right)(x)=\sin (\arcsin x)$

Aucune des fonctions $f \circ f^{-1}$ et $f^{-1} \circ f$ n'est égale à la fonction $x \mapsto x$ de $\mathbb{R}$ dans $\mathbb{R}$. Les courbes représentatives de ces deux fonctions sont nettement distinctes. 


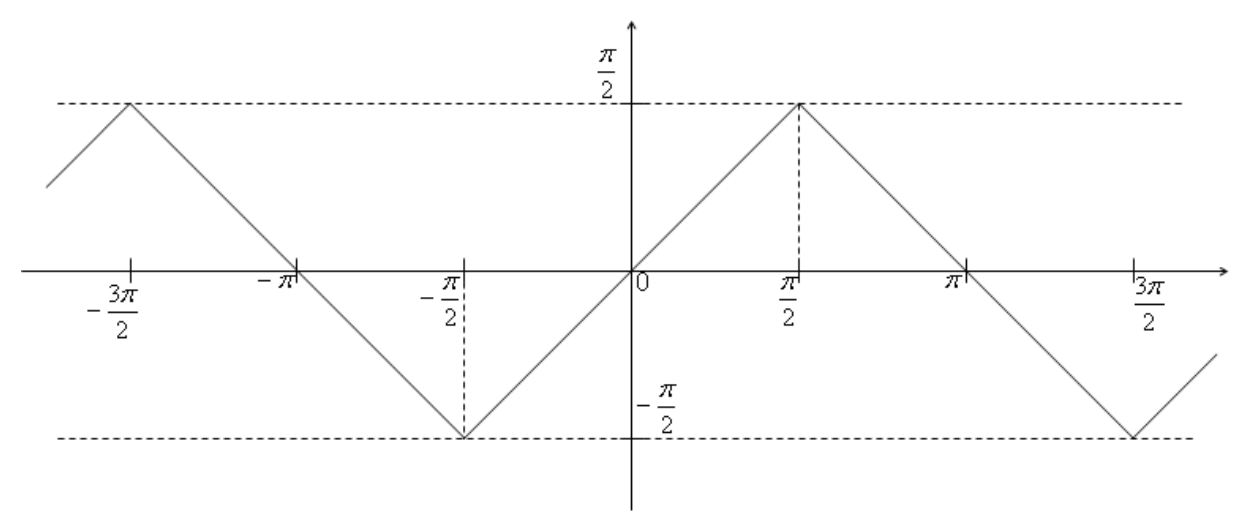

Figure 3. $\left(f^{-1} \circ f\right)(x)=\arcsin (\sin x)$

\subsection{Difficultés liées à la notion de fonction réciproque : des raisons possibles}

Nous proposons dans ce paragraphe des hypothèses pouvant expliquer la difficulté liée à la notion de fonction réciproque, surtout dans le cas où une restriction de la fonction initiale est nécessaire. Ces justifications éventuelles reposent sur les réactions de nombreux élèves de niveau $\mathrm{Bac}+1$, interrogés par André Antibi à l'université et en classes de mathématiques supérieures, durant une vingtaine d'années, de 1985 à 2005.

\subsubsection{Difficulté liée à la notion de convention}

Comme on l'a vu précédemment, la plupart des fonctions réciproques étudiées s'appuient préalablement sur une restriction de l'ensemble de définition de la fonction. Cette restriction arbitraire peut être source d'obstacle car son caractère artificiel peut dérouter certains élèves; surtout ceux qui veulent tout comprendre. Or dans ce cas il n'y a rien à comprendre réellement : on décide lors de cette étude de ne considérer qu'une certaine restriction de la fonction. Graphiquement, cela revient à ne considérer qu'une portion de la courbe représentative de la fonction initiale.

Ainsi par exemple, pour la fonction $f: x \mapsto x^{2}$, on ne considère que la partie «droite» de la courbe. Notons qu'il y a bien d'autres manières de définir une fonction réciproque de cette fonction $f$ : on pourrait se restreindre à la partie « gauche » de la courbe (en pointillés ci-contre), ou plus généralement à toute portion de courbe où la fonction est strictement monotone. On peut considérer 


$$
\text { "murillo" — 2008/9/1 - 17:25 — page } 57 \text { — \#15 }
$$

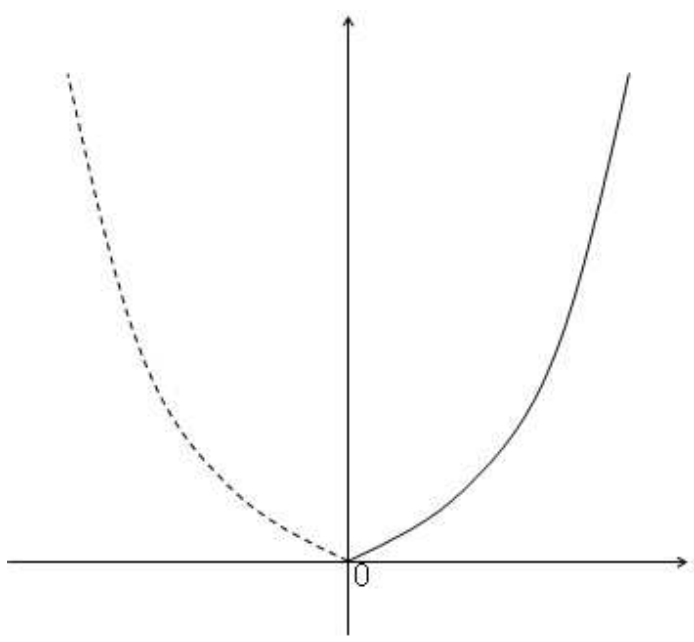

Figure 4

qu'il est assez naturel de choisir les «plus grands» intervalles possibles où il en est ainsi. Mais pourquoi conserver la partie «droite» de la courbe, et non pas la partie «gauche»? Ce choix est purement conventionnel.

\subsubsection{Difficulté liée au vocabulaire, aux notations}

Nous illustrons ce point sur deux exemples.

Fonction racine carrée $x \mapsto \sqrt{x}$

Avec la convention rappelée ci-dessus, $\sqrt{4}=2$ (et non pas -2 , ni \pm 2 ). Or l'équation $x^{2}=4$ admet deux racines : 2 et -2 .

Ainsi, la racine carrée de 4 est égale à 2 ; mais l'équation $x^{2}=4$ admet deux racines. Dans deux situations voisines, le mot «racine» a deux significations distinctes.

\section{Fonction «Arcsinus 》 $x \mapsto \arcsin x$}

L'origine de la terminologie «Arcsinus » pourrait paraître naturelle : $y=$ $\arcsin x$ signifie que y est l'Arc dont le sinus est $x$. Or cette idée est fausse : il s'agit de l'arc compris entre $-\frac{\pi}{2}$ et $\frac{\pi}{2}$ dont le sinus est égal à $x$.

En d'autres termes, il ne s'agit plus de la même fonction que la fonction $f: x \mapsto \sin x$ initiale (il s'agit de sa restriction à $\left[-\frac{\pi}{2} ; \frac{\pi}{2}\right]$ ), mais la notation est identique. 


\subsubsection{Un obstacle nécessitant une dé-transposition}

Rappelons d'abord, succinctement, qu'un obstacle nécessitant une dé-transposition est un obstacle dont l'origine est le système éducatif lui-mème ${ }^{5}$.

Plus précisément, lorsque l'on définit la bijection réciproque d'une bijection $f$ de $E$ dans $F$, on s'appuie sur l'idée intuitive de « chemin inverse ».

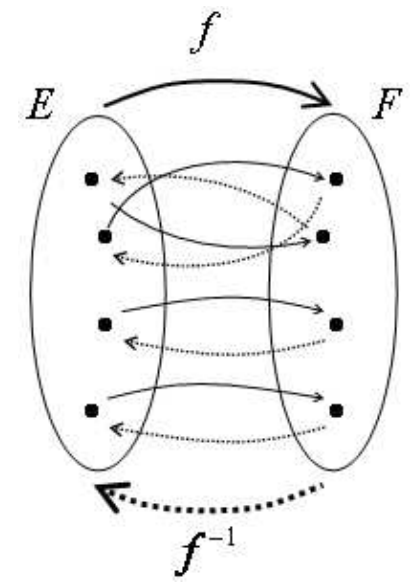

Figure 5

Par suite une idée forte est la suivante : $f \circ f^{-1}=I d_{F}$ et $f^{-1} \circ f=I d_{E}$ (il s'agit en quelque sorte d'un « aller-retour »).

Cette idée essentielle, vraie lorsque $f$ est une « vraie » bijection (sans restreindre son ensemble de définition) n'est plus vraie lorsqu'une restriction préalable est nécessaire. Ainsi,

$-(\sqrt{x})^{2}=x$ pour toute valeur de $x$ dans son champ de validité $(x \geq 0)$. Mais la propriété $《 \sqrt{x^{2}}=x »$ n'est pas toujours vraie dans son champ de validité $\mathbb{R}$ (elle n'est vraie que lorsque $x$ est positif).

- $\sin (\arcsin x)$ pour toute valeur de $x$ dans son champ de validité $([-1 ; 1])$. Mais la propriété « $\arcsin (\sin x) »$ n'est pas toujours vraie dans son champ de validité $\mathbb{R}$ (elle n'est vraie que lorsque $x$ appartient à $\left[-\frac{\pi}{2} ; \frac{\pi}{2}\right]$ ).

$\underline{\text { Remarque }}$ : les considérations possibles proposées ci-dessus $(2.4 .1 ; 2.4 .2$ et 2.4.3) se réfèrent au principe suivant : «Dans des conditions normales, lorsqu'une

5. Voir A. Antibi et G. Brousseau (2000 et 2002). 
majorité d'élèves éprouvent des difficultés, c'est le système éducatif qui en est responsable »(Antibi, 2003).

\section{Des solutions possibles}

3.1. Comment aider l'él'eve à surmonter les obstacles décrits ci-dessus?

Tout d'abord, il est utile que l'enseignant ait conscience des raisons possibles des difficultés rencontrées par les élèves. Chaque professeur, en fonction de sa sensibilité, peut alors utiliser des moyens pour y remédier.

Nous nous contentons ici de mentionner quelques pistes possibles :

\section{Notion de convention}

On pourrait insister sur le fait que d'autres choix seraient possibles; mais que la restriction choisie arbitrairement s'appuie sur deux points :

- «préférence» pour des intervalles centrés en 0 , les «plus grands » possibles : c'est le cas par exemple pour $\arcsin x$.

- Lorsqu'il est impossible de choisir un intervalle centré en 0 , «préférence » pour les nombres positifs : c'est le cas par exemple pour $\sqrt{x}$.

\section{Vocabulaire}

Pour la fonction «Arcsinus », par exemple, on pourrait utiliser la notation 《arcsin $/\left[-\frac{\pi}{2} ; \frac{\pi}{2}\right] »$, ou encore pour simplifier : $《 \arcsin / I »$, en ayant préalablement posé $I=\left[-\frac{\pi}{2} ; \frac{\pi}{2}\right]$.

\section{Obstacle nécessitant une dé-transposition}

Dans le cas d'un obstacle nécessitant une dé-transposition, nous suggérons fortement le point suivant : il convient de gérer avec beaucoup de soin une telle situation, en ne se contentant pas de signaler aux élèves de «faire attention ». On pourrait par exemple proposer à l'élève une activité lui permettant de prendre conscience par lui-mème qu'il y a un problème.

\subsection{Utilité d'enseigner de telles fonctions?}

On peut s'interroger sur l'opportunité d'introduire les fonctions réciproques nécessitant une restriction préalable de l'ensemble de définition de la fonction initiale. L'introduction de telles fonctions dans les programmes scolaires est vraisemblablement une conséquence de l'enseignement des mathématiques modernes. 


$$
\text { "murillo" — 2008/9/1 — 17:25 — page } 60-\# 18
$$

Dans ce contexte, la notion d'application et d'ensemble de définition associé jouaient un rôle important ${ }^{6}$. Or, les mathématiques modernes ont disparu des programmes de l'enseignement secondaire français, et on insiste beaucoup moins sur la théorie des ensembles.

Quel danger y aurait-il à écrire $\sqrt{4}= \pm 2$ ? Ou encore $\arcsin x=x+2 k \pi$ $(k \in \mathbb{Z})$ ?

Il ne s'agirait plus alors de vraies fonctions, mais on éviterait ainsi la plupart des obstacles décrits précédemment, en s'appuyant sur la notion de chemin inverse, naturelle et qui a du sens. Il y a un demi-siècle environ, on parlait de fonctions multiformes dans ce cas.

\section{Références}

[1] A. Antibi, Etude sur l'enseignement de méthodes de démonstration. Enseignement de la notion de limite : réflexions, propositions, thèse, Université de Toulouse, 1988.

[2] A. Antibi et G. Brousseau, La dé-transposition de connaissances scolaires, Recherche en Didactique des Mathématiques 20, no. 1, 2000, 7-40.

[3] A. Antibi et G. Brousseau, Vers l'ingénierie de la dé-transposition, Didactiques des disciplines scientifiques et technologiques : concepts et méthodes 8, 2002, 45-57.

[4] A. Antibi, La constante Macabre, Math'Adore, 2003.

[5] M. Artigue, Réformes et contre-réformes de l'enseignement de l'analyse au lycée (1902-1994), in: Les sciences au lycée - un siècle de réformes des mathématiques et de la physique en France et à l'étranger, (B. Belhoste et al., eds.), Ed. Vuibert, Paris, 1996, 197-217.

[6] M. Artigue, Evolutions et perspectives de l'enseignement de l'analyse au lycée, L'ouvert 107, 2003, 1-18.

[7] I. Bayazit et E. Gray, Understanding inverse functions: the relationship between teaching practice and student learning, Proceedings of the $28^{\text {th }}$ Conference of the International Group for the Psychology of Mathematics Education 2, 2004, 103-110.

[8] G. Brousseau, Théorie de situations didactiques, Ed. La Pensée Sauvage, Grenoble, 1998.

[9] Y. Chevallard, Cours 3 : Ecologie et Régulation; Thème 1 : Les praxéologies didactiques, in: Actes de la $11^{e}$ Ecole d'été de didactique des mathématiques, (J. L. Dorier et al., eds.), La Pensée Sauvage Ed., Grenoble, 2002.

6. Mème à l'époque des mathématiques modernes, la notion de fonction, et non d'application, était considérée comme mieux adaptée à certains problèmes, les limites par exemple (Antibi, 1988). 


$$
\text { "murillo" — 2008/9/1 — 17:25 — page } 61 \text { — \#19 }
$$

[10] R. Douady, Jeux de cadres et dialectique outil-objet, Recherche en Didactique des Mathématiques 7, no. 2, 1986, 5-31.

[11] R. Even, The inverse function: Prospective teachers' use of "undoing", International Journal of Mathematical Education in Science and Technology 23, no. 4, 1992, $557-562$.

[12] J. Itard, Essais d'histoire des mathématiques, Librairie Scientifique et Technique, Paris, 1984.

[13] C. Lucus, Is subject matter knowledge affected by experience? The case of composition of functions, Proceedings of the $30^{\text {th }}$ Conference of the International Group for the Psychology of Mathematics Education 4, 2006, 97-104.

[14] A. Robert, Outils d'analyse des contenus mathématiques à enseigner au lycée et à l'université, Recherches en Didactique des Mathématiques 18, no. 2, 1998, 139-190.

[15] M. Rogalski, Carrefours entre analyse, algèbre et géométrie, Ed. Ellipses, Paris, 2001.

[16] L. Schwartz, Cours d'analyse, Ed. Hermann, Paris, 1981.

[17] P. Trabal, La violence de l'enseignement des mathématiques et des sciences : une autre approche de la sociologie des sciences, Ed. L'Harmattan, Montréal, 1997.

[18] D. Vidakovic, Learning the concept of inverse function, Journal of Computers in Mathematics and Science Teaching 15, no. 3, 1996, 295-318.

[19] A. P. Yousschkevitch, Le concept de fonction jusqu'au milieu du XIX ${ }^{\mathrm{e}}$ siècle in Fragments d'histoire des mathématiques, Brochure APMEP, no. 41, 1981, 7-68.

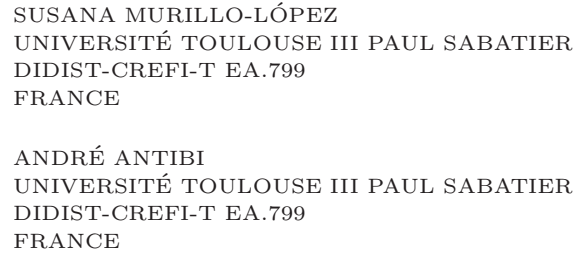

(Received July, 2007) 